\title{
Local Inflammatory Reaction to Vancomycin-Laden Bone Cement
}

\author{
Heather Young, Whitney Miller, Michael Wilson, ${ }^{2}$ Cyril Mauffrey, ${ }^{3}$ and E. Mark Hammerberg ${ }^{3}$
}

\begin{abstract}
Background: Antibiotic-laden bone cement is used commonly for fixation of primary hip arthroplasty and in the creation of spacers for treatment of orthopedic infections. We report an adverse event associated with the use of a vancomycin-laden bone cement spacer for treatment of a total knee arthroplasty infection.

Case Presentation: A 60-year old Hispanic male developed chronic total knee arthroplasty infection caused by coagulase-negative staphylococci and anaerobic bacteria. He received systemic vancomycin and a vancomycinladen cement spacer. During treatment, the patient developed a macular rash that resolved with discontinuation of intravenous vancomycin. He completed a six-week course of antibiotics uneventfully. When the patient returned to the operating room for revision arthroplasty, substantial local inflammation was found in the frozen sections. The vancomycin-laden cement spacer was removed and replaced with a spacer that did not contain vancomycin. No organisms were found on either routine microbiologic culture or broad-range polymerasechain reaction studies. The Naranjo nomogram showed a "probable" adverse drug reaction to vancomycin cement. The patient received six more weeks of broad-spectrum antibiotics and eventually underwent successful revision arthroplasty.

Conclusion: We believe that this patient had systemic and local allergic reactions to vancomycin. Because antibiotic-laden bone cement and other topical antibiotic preparations are used commonly in the treatment of surgical infections, we encourage providers to be aware of the possible adverse effects.
\end{abstract}

\begin{abstract}
A NTIBIOTIC-LADEN BONE CEMENT (ALBC) is used commonly for fixation of primary hip arthroplasty and in the creation of spacers for treatment of orthopedic infections [1]. Because of its antimicrobial spectrum and heat-stable properties, vancomycin is among the most common antibiotics used in ALBC. Vancomycin is a glycopeptide that is effective in the treatment of gram positive bacteria. Adverse events after systemic administration may occur including red man syndrome, urticaria, macular rashes, exfoliative dermatitis, eosinophilia, vasculitis, anaphylaxis, ototoxicity, nephrotoxicity, and hematologic toxicity [2]. High-dose vancomycin ( $>2 \mathrm{~g}$ vancomycin per $40 \mathrm{~g}$ packet of cement) is considered to be a safe means of local antibiotic delivery $[3,4]$. We report an adverse event associated with the use of vancomycin ALBC spacer for treatment of a total knee arthroplasty (TKA) surgical site infection.
\end{abstract}

\section{Case Presentation}

A 60-year old male presented to the emergency department after falling because he was unable to bear weight on his left knee. He reported having several months of pain and swelling in the left knee preceding the fall. The patient had undergone a right TKA 10 years prior to presentation and a left TKA two years prior to presentation for degenerative joint disease. In addition to osteoarthritis, his past medical history was significant for type 2 diabetes mellitus, hyperlipidemia, and gastroesophageal reflux disease. On review of systems, the patient reported fatigue and sweating. He was originally from Mexico but had resided in the United States for several years. He had a remote history of alcohol and tobacco abuse and no history of illicit drug use.

Physical examination revealed a well-appearing male with normal vital signs. An effusion was present in the left knee

\footnotetext{
${ }^{1}$ Division of Infectious Diseases, ${ }^{2}$ Department of Pathology, ${ }^{3}$ Department of Orthopedic Surgery, Denver Health Medical Center and University of Colorado Hospital, Denver, Colorado.

(C) Heather Young et al. 2016; Published by Mary Ann Liebert, Inc. This Open Access article is distributed under the terms of the Creative Commons License (http://creativecommons.org/licenses/by/4.0), which permits unrestricted use, distribution, and reproduction in any medium, provided the original work is properly credited.
} 
and edema in the left distal femur. The left TKA incision was well healed. The patient had intact neurovascular examination. Laboratory studies included $10.9 \times 10^{3}$ leukocytes per microliter, hematocrit $41.1 \%, 303 \times 10^{3}$ platelets per microliter, erythrocyte sedimentation rate (ESR) $42 \mathrm{~mm} / \mathrm{h}$ (reference range, $<20 \mathrm{~mm} / \mathrm{h}$ ), and C-reactive protein (CRP) $100 \mathrm{mg} / \mathrm{L}$ (reference range, $0-10 \mathrm{mg} / \mathrm{L}$ ). Synovial fluid was obtained and cell counts revealed more than $32,000 \times 10^{3}$ leukocytes per microliter with $99 \%$ polymononuclear cells (PMNs). Intra-cellular bacteria were observed on microscopic examination, and no crystals were detected. Plain film radiograph of the left leg showed a large effusion of the knee and lucency around the femoral component of the TKA.

The patient was taken to the operating room for presumed septic arthroplasty. Chronic synovitis was encountered without frank purulence. Both the femoral and tibial components of the TKA were removed. An articulating spacer was created containing a $8 \mathrm{~g}$ of vancomycin in $120 \mathrm{~g}$ of cement. Routine cultures of the femur were submitted for aerobic, anaerobic, and fungal pathogens were obtained. Cultures grew Staphylococcus epidermidis, S. capitis, and mixed anaerobic bacteria. The patient was discharged from the hospital receiving intravenous vancomycin and oral metronidazole.

The patient responded well to the treatment course with no drainage from his surgical incisions and markedly decreased pain. However, approximately three weeks into antibiotic therapy, the patient developed a pruritic, erythematous, macular rash predominantly on the trunk. Vancomycin was discontinued and the rash subsided. The patient completed the remainder of his six-week course of antibiotics by taking intravenous daptomycin and oral metronidazole. At the end of therapy, CRP normalized to $<10 \mathrm{mg} / \mathrm{L}$.

Two weeks after discontinuing antibiotics, the patient returned to the operating room for revision arthroplasty. Although no purulence was found, frozen sections revealed more than five PMNs per high-power field in four of the five tissue samples, and one of the five samples had more than 10 PMNs per high-power field. Although infection was considered the most worrisome cause of the inflammation, local drug reaction caused by vancomycin ALBC was also considered as a possibility. The Naranjo nomogram [5] was applied to the patient's case and the results were "probable", adverse drug reaction ( 2 points for the adverse event appearing after the drug was administered; 1 point for each of conclusive case reports on this reaction, similar reaction to the same drug with a previous exposure, and objective evidence of the adverse event; -1 point for alternative causes that could have caused the reaction).

Instead of revision arthroplasty, the vancomycin ALBC spacer was removed and a new articulating ALBC spacer was placed that contained $4 \mathrm{~g}$ of daptomycin and $2.4 \mathrm{~g}$ of tobramycin in $80 \mathrm{~g}$ of cement. Vancomycin was not used to in this spacer because of the documented vancomycin allergy. All standard intra-operative cultures were negative, and broad-spectrum polymerase chain reaction (PCR), performed at the University of Washington reference laboratory, also failed to detect bacteria.

The patient was treated again with antimicrobial therapy. He received six additional weeks of intravenous daptomycin and oral levofloxacin. His post-operative physical examination again normalized quickly. He was taken to the operating room for revision arthroplasty two weeks after discontinuation of antibiotics for a second time; this time, all frozen sections revealed more than five PMNs per high-power field. The TKA was implanted successfully and he had no signs of infection in three months of follow-up.

\section{Discussion and Literature Review}

Adverse events associated with ALBC are reported widely in the literature. The rate of acute kidney injury (AKI) after ALBC spacer placement is reported at approximately $5 \%$ (range, 2\%-17\%), although the true rate may be greater because of under-reporting [6-9]. Because most ALBC contains both an aminoglycoside (i.e., tobramycin or gentamicin) and vancomycin, it is difficult to determine which of these antibiotics contributes the greater effect to the development of AKI. In addition to AKI, serious cutaneous reactions have been attributed to vancomycin ALBC. Williams et al. [10] reported the development of a desquamating rash after implantation of vancomycin ALBC in a patient with a history of vancomycin-induced Stevens-Johnson syndrome.

Vancomycin and aminoglycosides have been shown to elute from ALBC at high quantities, making ALBC an attractive means of local antibiotic delivery $[11,12]$. However, these high local concentrations place patients at risk for local tissue reactions such as those described in the case report above.

\section{Conclusion}

The histologic findings associated with hypersensitivity to ALBC have not been well described. The present case demonstrates findings consistent with acute inflammation, with neutrophil counts exceeding five PMNs per high-power field. This is perplexing because neutrophil counts are used typically to exclude persistent infection at the time of revision surgery. In the present case, the local inflammatory response appears to be related to vancomycin inclusion in ALBC, as infection has been ruled out by negative culture results and by broad-range PCR. Furthermore, we noted resolution of inflammation after vancomycin was excluded from the subsequent ALBC spacer. Further studies on the benefits and toxicities of ALBC are required to define further its optimal use.

\section{Author Disclosure Statement}

No competing financial interests exist

\section{References}

1. Hake ME, Young H, Hak DJ, et al. Local antibiotic therapy strategies in orthopaedic trauma: Practical tips and tricks and review of the literature. Injury 2015;46:1447-1456.

2. Bruniera F, Ferreira F, Saviolli L, et al. The use of vancomycin with its therapeutic and adverse effects: A review. Eur Rev Med Pharmacol Sci 2015;19:694-700.

3. Osmon DR, Berbari EF, Berendt AR, et al. Diagnosis and management of prosthetic joint infection: Clinical practice guidelines by the Infectious Diseases Society of America. Clin Infect Dis 2013;56:e1-e25.

4. Parvizi J, Adeli B, Zmistowski B, et al. Management of periprosthetic joint infection: The current knowledge: AAOS exhibit selection. J Bone Joint Surg Am 2012;94:e104.

5. Michel DJ, Knodel LC. Comparison of three algorithms used to evaluate adverse drug reactions. Am J Hosp Pharm 1986;43:1709-1714. 
Address correspondence to:

Dr. Heather Young

660 Bannock Street

MC 4000

Denver, CO 80204

E-mail: heather.young2@dhha.org hip joint infections. Int J Med Sci 2009;6:265-273.

8. Luu A, Syed F, Raman G, et al. Two-stage arthroplasty for prosthetic joint infection: A systematic review of acute kidney injury, systemic toxicity and infection control. J Arthroplasty 2013;28:1490-1488.

9. Armaghani SJ, Menge TJ, Lovejoy SA, et al. Safety of topical vancomycin for pediatric spinal deformity: Nontoxic serum levels with supratherapeutic drain levels. Spine 2014;39:1683-1687.

10. Williams B, Hanson A, Sha B. Diffuse desquamating rash following exposure to vancomycin-impregnated bone cement. Ann Pharmacother 2014;48:1061-1065.

11. Niikura T, Lee SY, Iwakura T, et al. Antibiotic-impregnated calcium phosphate cement as part of a comprehensive treatment for patients with established orthopaedic infection. J Orthop Sci 2016;21:539-545.

12. Hinarejos P, Guirro P, Puig-Verdie L, et al. Use of antibiotic-loaded cement in total knee arthroplasty. World J Orthop 2015;6:877-885.

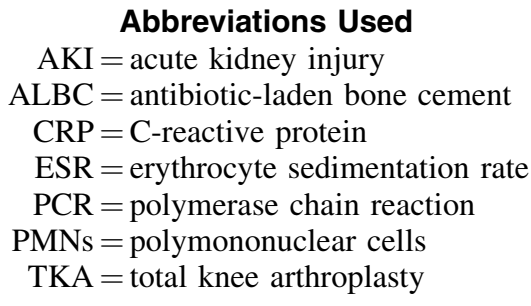

Cite this article as: Young $\mathrm{H}$, Miller $\mathrm{W}$, Wilson M, Mauffrey C, Hammerberg EM (2016) Local inflammatory reaction to vancomycin antibiotic-laden bone cement. Surgical Infections Case Reports 1:1, 97-99, DOI: $10.1089 /$ crsi.2016.0023 